Cahiers de Narratologie

Nouvelles frontières du récit. Au-delà de l'opposition entre factuel et fictionnel

\title{
Cinéma : lieu de la construction de la mémoire collective
}

Le cas du cinéma chinois de la Cinquième Génération des années 1980

Wenjun Deng

\section{OpenEdition}

\section{Journals}

\section{Electronic version}

URL: http://journals.openedition.org/narratologie/6896

DOI: $10.4000 /$ narratologie.6896

ISSN: 1765-307X

Publisher

LIRCES

\section{Electronic reference}

Wenjun Deng, «Cinéma : lieu de la construction de la mémoire collective », Cahiers de Narratologie

[Online], 26 | 2014, Online since 11 September 2014, connection on 19 April 2019. URL : http:// journals.openedition.org/narratologie/6896 ; DOI : 10.4000/narratologie.6896

This text was automatically generated on 19 April 2019

Article L.111-1 du Code de la propriété intellectuelle. 


\title{
Cinéma : lieu de la construction de la mémoire collective
}

Le cas du cinéma chinois de la Cinquième Génération des années 1980

\author{
Wenjun Deng
}

Le cinéma est considéré comme un lieu privilégié de la mémoire collective, dont la fonction consiste à transmettre les souvenirs du passé à des contemporains et des générations futures et à permettre l'affirmation d'une identité collective. Cet article sera consacré, en mettant en relation mémoire et identité, à aborder la construction de la mémoire collective de la Révolution culturelle dans le cinéma chinois de la Cinquième Génération des années 1980. La Cinquième Génération désigne un groupe spécifique de cinéastes chinois qui ont consacré leur jeunesse aux camps de travaux forcés à la campagne, suite à l'appel à la rééducation lancé par Mao durant la Révolution culturelle ; ce groupe s'engage dans le métier du cinéma au début des années 1980. Ces cinéastes construisent une mémoire collective de la Révolution culturelle et ajoutent l'évocation de l'expérience personnelle au récit collectif dans leurs films. La question de la manière dont le cinéma constitue un passage des formes individuelles de la mémoire et de l'identité à des formes collectives sera au cœur de la présente réflexion.

mémoire collective, identité, cinéma, la Cinquième Génération, individu

L'historien du cinéma italien Gian Piero Brunetta développe l'idée que le cinéma se constitue en lieu privilégié de mémoire en indiquant que parmi tous les lieux de mémoire, le cinéma aspire tout de suite à devenir et, de fait, devient le métronome, le traducteur et l'interprète des temps et des rythmes sociaux nouveaux, mais aussi l'archive vivante de la mémoire de la vie de la planète ${ }^{1}$. Le cinéaste utilise sa caméra pour enregistrer le temps présent et aussi pour revenir sur le passé ; le cinéma nous aide ainsi à comprendre ce passé en offrant une vision du monde et modifie également notre conception de la mémoire. D'après Gian Piero Brunetta, les films produits en Italie peuvent être considérés dans leur totalité comme "récit de l'histoire et mémoire collective ». Dans tous ces films, on peut reconnaître, écrit-il, une chanson de geste hyperbolique, et dans les aventures infinies du quotidien, un point de fixation, de légitimation et de célébration de l'histoire 
et de la biographie collective de millions de personnes sans histoire. Les sources cinématographiques, dans leur accumulation en ordre dispersé, dans leur défilement qui suit en une symbiose parfaite le cours de toutes les chronologies et achronies du siècle, confirment leur vocation naturelle à se constituer en lieu privilégié de la mémoire collective ${ }^{2}$. Le cinéma n'a pas cependant de dimension physique. C'est plutôt un lieu de mémoire imaginaire et virtuel dont la substance est construite à travers la production de signifiants cinématographiques et la projection sur l'écran. Le matériau filmique s'incorpore ainsi à la mémoire sociale. La fonction de ce lieu de mémoire consiste ainsi à transmettre les souvenirs du passé à des contemporains et des générations futures et à permettre l'affirmation d'une identité collective. Le développement du concept de Brunetta permet de renvoyer au cinéma chinois qui est doté d'une identité fortement enracinée dans sa propre histoire et culture. Cet article sera consacré à étudier la construction de la mémoire collective dans le cinéma de la Cinquième Génération dans les années 1980.

L'idée de classifier les réalisateurs chinois selon le critère de " génération » en fonction de la chronologie de leur entrée en scène est originairement introduite par les chercheurs occidentaux, tel que Jean Michel Frodon, pour décrire l'évolution du septième art en Chine. Dans son usage conventionnel, la notion de « Cinquième Génération » désigne un groupe de jeunes cinéastes chinois diplômés en 1982 de l'Institut du cinéma de Pékin et s'y étant inscrit en tant que première promotion depuis la réouverture de l'Institut en 1978, suite à une fermeture de dix ans pendant la Révolution culturelle. La Révolution culturelle fut un événement traumatisant dans l'histoire chinoise, ayant plongé d'innombrables Chinois de l'époque dans une immense misère et paralysé des activités dans tous les secteurs de la société chinoise pendant dix ans : les universités et les écoles furent fermées ; il n'y avait pas de livres à lire car ceux-ci furent brûlés; la quasi-totalité des fonctionnaires, cadres, éducateurs, intellectuels sur le territoire chinois ont été humiliés publiquement, parmi lesquels beaucoup ont subi une persécution mortelle et ont vu leurs familles ruinées et dispersées. Près de dix-sept millions de jeunes citadins ont été exilés sous l'impulsion de Mao dans les régions rurales et forcés de travailler aux champs pour être rééduqués auprès des paysans durant le mouvement d'envoi des jeunes instruits à la campagne qui fut déclenché en 1968 et s'acheva en 1980. Confrontés à de dures réalités dans les régions reculées et pauvres et souffrant d'une double pauvreté, matérielle et spirituelle, les jeunes instruits perdirent leurs illusions et l'opportunité de poursuivre leurs études, et sombrèrent dans le désespoir. Ainsi est engendrée par la Révolution culturelle la " génération perdue ${ }^{3}$ ", dont les cinéastes de la Cinquième Génération font partie.

Chen Kaige est considéré comme le porte-parole de cette génération de cinéastes chinois alors que les cinéastes représentatifs en sont Zhang Junzhao, Tian Zhuangzhuang, Huang Jianxin, Wu Ziniu, Zhang Yimou, Hu Mei ${ }^{4}$. Etant entrée en scène au début des années quatre-vingt, la Cinquième Génération est confrontée à la rupture culturelle et identitaire de la société chinoise, rupture qui peut être interprété comme le résultat de la destruction des biens culturels et du mépris envers les valeurs traditionnelles, en vogue pendant la Révolution culturelle. Suite aux fractures et blessures causées par la Révolution culturelle, surmonter la rupture culturelle et identitaire et renouer le passé et le futur s'imposent comme des enjeux vitaux à la société chinoise des années 1980. Les cinéastes de la Cinquième Génération qui entrent en scène au bon moment assument consciemment le rôle de transmission de la mémoire et de la culture traditionnelle et 
s'engagent à construire une nouvelle identité collective à l'aide de la mise en scène. Nourris de l'expérience de la Révolution culturelle, les cinéastes de cette génération tournent leur caméra vers la tradition, la culture et l'individu dans la société et proposent une vision de cette histoire traumatisante de manière métaphorique et allégorique. Comprendre de quelle manière le cinéma se constitue comme un passage des formes individuelles de la mémoire et de l'identité à des formes collectives est au cœur de nos interrogations. Pour ce faire, nous mettrons en relation les concepts de mémoire et d'identité en nous référant à Joël Candau.

La Cinquième Génération : entre mémoire et identité

La mémoire et l'identité entretiennent une relation intrinsèque et indissoluble. Cette relation dialectique est développée davantage par Joël Candau dans son livre Mémoire et identités, dans lequel il souligne qu'il devient difficile d'accorder la prééminence à l'une ou à l'autre dès que l'on considère l'homme en société. En fait, mémoire et identité se compénètrent. Elles se renforcent mutuellement, depuis le moment de leur émergence jusqu'à leur inéluctable dissolution. Il n'y a pas de quête identitaire sans mémoire et, inversement, la quête mémorielle est toujours accompagnée d'un sentiment d'identité, au moins individuelle ${ }^{6}$. Constituant une des activités fondamentales des sociétés d'aujourd'hui, la quête de l'identité ne peut pas s'effectuer sans s'appuyer sur la mémoire de même que la mémoire ne peut pas être traitée sans tenir compte de l'identité. La mémoire et l'identité s'incorporent et s'assimilent l'une l'autre, se fondent mutuellement pour produire une trajectoire individuelle et le parcours historique d'une nation. Du coup, il est pertinent de retracer le parcours individuel des cinéastes de la Cinquième Génération pendant la Révolution culturelle afin de mettre en exergue une quête mémorielle de l'histoire et les représentations de leur mémoire identitaire dans leur cinéma. D'après Joël Candau, si la mémoire est « générative » de l'identité, dans le sens où elle participe à sa construction, celle-ci, en retour, façonne des prédispositions qui vont conduire l'individu à « incorporer » certains aspects particuliers du passé, à faire des choix mémoriels ${ }^{7}$. Le cinéma nous aide à comprendre le passé en se faisant vision du monde et modifie parfois notre conception de la mémoire. Chaque cinéaste possède sa mémoire individuelle de la Révolution culturelle, qui vient nourrir sa création cinématographique et lui permet de proposer sa vision de tel ou tel aspect du passé auquel il est sensibilisé. Cette mémoire individuelle prédispose effectivement l'identité de l'individu, dont la formation est étroitement liée aux bouleversements sociaux pendant la Révolution culturelle.

Au sein de l'existence personnelle des réalisateurs de la Cinquième Génération, la Révolution Culturelle constitue une expérience cruciale qui modifie désormais leur parcours et réédifie leur univers mental et leur vision du monde. C'est ce que Chen Kaige indique dans son autobiographie ${ }^{8}$, « à mon avis, la plupart de mes expériences de vie ont dérivé de cette époque-là [la Révolution culturelle]. Ce qui est le plus important, c'est le fait que cette révolution m'ait permis de me connaître. Se connaître soi-même c'est connaître le monde. Ce fut important pour le restant de ma vie de comprendre cela ${ }^{9}$ ». La plupart des cinéastes de la Cinquième Génération sont dotés d'une double identité dans la Révolution culturelle : Gardes rouges au début de l'éclatement de la Révolution culturelle et jeunes intellectuels durant le mouvement d'envoi des jeunes instruits à la campagne. L'expérience de Chen Kaige, le porte-parole de la Cinquième Génération, peut être considérée comme une allégorie de toute la génération de l'époque. C'est ce que le réalisateur affirme en parlant de son autobiographie, « elle [l'autobiographie] ne parle 
pas d'un jeune qui s'appelle Chen Kaige, mais de cette époque-là ${ }^{10} »$. Né dans une famille de cinéastes, le père de Chen Kaige était un réalisateur faisant partie de la Troisième Génération et sa mère une scénariste avant la Révolution culturelle. Dans son autobiographie, Chen Kaige rappelle son expérience, en tant que Garde rouge, d'avoir trahit et dénoncé son père accusé d'être un « contre-révolutionnaire » et de l'avoir humilié en public durant une réunion de critique pour tracer une ligne de démarcation nette avec son père. De plus, ses anciens amis ont fait irruption dans sa famille et détruit tout ce qu'ils considéraient comme objets féodaux. Toutes ces expériences douloureuses sont vécues comme une perte de dignité et constituent les souvenirs qui l'obsèdent. Par la suite, il est envoyé, comme la plupart de ses collègues, dans une région rurale et reculée dans la province du Yunnan et y consacre ses trois premières années de « rééducation » au travail d'abattage du bois dans une plantation de caoutchouc. Cette expérience de « rééducation » lui permet de nourrir sa réflexion sur le rapport entre l'individu et la collectivité et de prendre conscience des valeurs individuelles, méprisées et foulées aux pieds pendant la Révolution culturelle. En témoignant personnellement de l'existence misérable dans les régions rurales de " rééducation ", Chen Kaige, dans un premier temps, n'exorcise pas dans son cinéma de manière directe ses souvenirs de la Révolution culturelle, mais évoque plutôt cette expérience de manière métaphorique et surtout mène une réflexion profonde sur l'existence humaine (dans La Terre jaune), le rapport entre l'individu et la collectivité (dans La Grande parade) et l'individu sous l'oppression de l'institution (dans Le Roi des enfants).

L'expérience de Tian Zhuangzhuang est semblable à celle de Chen Kaige: ils ont en commun une expérience douloureuse de dénonciation de leurs parents en public pendant la Révolution culturelle. Tian est né dans une famille d'artistes : son père Tian Fang et sa mère Yu Lan étaient tous deux des acteurs reconnus en Chine avant la Révolution culturelle. Tian passe ses années de jeune instruit dans la province du Jilin. Il révèle dès le début de sa carrière son intérêt particulier pour le thème des minorités ethniques et sa sensibilité aux rituels et aux rythmes de la société tribale ainsi qu'à l'existence misérable des habitants minoritaires en tournant La loi du terrain de chasse (Tian Zhuangzhuang, 1985) et Le voleur de chevaux (Tian Zhuangzhuang, 1986). D'après Tian, ces deux films peuvent sembler traiter des minorités nationales, mais ils se réfèrent en réalité au destin de la nation chinoise dans son ensemble ${ }^{11}$. Loin d'être une création improvisée, ces films ont un lien étroit avec l'expérience de jeunesse du réalisateur dont l'identité de jeune instruit exerce une influence profonde.

D’après Tony Rayns, Wu Ziniu, un des cinéastes de la Cinquième Génération, est moins traumatisé par la Révolution culturelle que ses autres contemporains qui viennent des grandes villes, tels que Chen Kaige et Tian Zhuangzhuang, et ses films sont relativement moins marqués par les traces de ses expériences à la campagne. Depuis son premier long métrage, Bloodshed in the Dark Valley (Wu Ziniu, 1984), il révèle sa préférence pour le sujet de la guerre. Le film, dit Tony Rayns, établit concrètement la guerre comme un milieu naturel ; comme Sam Fuller, il maintient une attitude d'anti-guerre irréprochable, tout en considérant le « drame » comme synonyme de « conflit » de manière à rendre le choix de son sujet de guerre et les métaphores inévitables ${ }^{12}$. Cependant, ni l'expérience de Wu Ziniu ni son cinéma ne sont aussi simples que l'explique Tony Rayns. Dans un entretien ${ }^{13}$, le cinéaste évoque ses souvenirs traumatisants de la Révolution culturelle : sa sœur devient folle suite à une série de malheurs insupportables survenant dans la famille lors du déclenchement de la Révolution culturelle, ce qui constitue un coup fatal pour Wu, qui 
affirmera : " rien n'est aussi choquant pour moi ». En outre, il témoigne d'une scène de lutte armée entre deux factions de Gardes rouges ayant eu lieu à l'été 1967 à Leshan, dans laquelle un jeune Garde rouge fut tué dans une pluie de balles sur un grand bâtiment alors qu'il voulait illuminer le champ de bataille avec un projecteur. Ces souvenirs obsèdent le cinéaste et suscitent en lui une réflexion profonde sur la vie et le destin individuel : la belle vie est tellement fragile face à la réalité cruelle, la destruction et la cruauté de la vie, la subversion et l'affolement, l'individu se sacrifie à l'histoire et à la politique. « Il faut retrouver le soi-même ». Mais comment retrouver la dignité de la vie dans la cruauté de la réalité ${ }^{14}$. Le destin individuel est au cœur de la réflexion que le cinéaste mène dans ses films au sujet de la guerre, tels que The Last Day of Winter et Evening Bell (Wu Ziniu, 1988).

Comme la plupart de ses contemporains, Zhang Yimou passe ses années de jeune instruit dans un village de la province du Shannxi. Pour Zhang Yimou, sa mémoire personnelle ne peut être reconstruite sans s'appuyer sur la mémoire familiale. Comme le note Joël Candau, la mémoire et l'identité personnelle doivent toujours composer avec la mémoire familiale, mémoire forte, qui exerce son pouvoir bien au-delà de liens apparemment distendus ${ }^{15}$. Zhang Yimou évoque dans sa biographie le fait que son père et ses oncles sont tous diplômés de l'Académie militaire de Huangpu ${ }^{16}$. L'un de ses deux oncles se rend à Taiwan alors que l'autre est accusé d'être un « traitre caché » après avoir été tué dans les années 1940 par le Parti nationaliste chinois ${ }^{17}$ alors qu'il voulait se mettre sous la protection du communisme à Yan'an ; son père est accusé d'être un contrerévolutionnaire durant la Révolution culturelle. Élevé dans une famille obsédée par toute sorte de « chefs d'accusation » politiques, le sentiment de répression du réalisateur est permanent. Ainsi, le désir d'émanciper l'individu constitue un thème récurrent dans ses films tels que Le Sorgho rouge (1988), Ju Dou (1990), Épouses et concubines (1991) dans lesquels ce désir d'émancipation est métaphorisé par un désir sensuel. Au lieu de contenir leur sentiment, les personnages de ses films, tels que Jiu'er, Judou ou encore Songlian luttent contre la répression et les contraintes des rites féodaux. Zhang Yimou met en évidence la passion individuelle et le désir sensuel enterrés par la culture officielle pendant la Révolution culturelle.

Le cinéma : un lieu où se rejoignent mémoire collective et individuelle

D’après Maurice Halbwachs, l'individu participerait à deux sortes de mémoires. D'une part, c'est dans le cadre de sa personnalité, ou de sa vie personnelle, que viendraient prendre place ses souvenirs alors qu'il serait capable, d'autre part, à certains moments de se comporter simplement comme le membre d'un groupe contribuant à évoquer et entretenir des souvenirs impersonnels, dans la mesure où ceux-ci intéressent le groupe ${ }^{18}$. Chaque cinéaste de la Cinquième Génération a son expérience et ses souvenirs personnels de la Révolution culturelle. Leur mémoire individuelle s'appuie sur la mémoire collective et se replace en elle pour préciser et même combler les lacunes de cette dernière, elle s'assimile et s'incorpore ainsi progressivement à la mémoire collective et contribue à la construction de cette dernière. C'est dans leur cinéma que la Cinquième Génération passe des formes individuelles de la mémoire et de l'identité à des formes collectives. En se référant de manière implicite ou métaphorique à l'expérience et aux souvenirs des cinéastes de la Révolution culturelle, le cinéma de la Cinquième Génération porte un discours à plusieurs niveaux: retour à la tradition et à la racine ; prise de conscience de la valeur individuelle ; réflexion sur le destin individuel.

Retour à la tradition et la racine 
En tant qu'anciens jeunes instruits faisant partie d'une génération sacrifiée qui s'est vue marginalisée par l'ordre urbain après des années de rééducation à la campagne, les cinéastes ont en commun la nécessité de reconstruire une identité profondément menacée pour redevenir eux-mêmes. À la différence de la génération précédente ayant eu recours à l'abréaction de souvenirs douloureux, cette génération de jeunes cinéastes tourne la page de l'histoire et fait preuve d'un intérêt et d'une attention particulière pour la tradition et les racines individuelles, afin de se retrouver soi-même, ce que souligne Joël Candau : la restauration de l'identité individuelle peut passer par l'abréaction de souvenirs douloureux ou traumatiques, préalablement censurés ou masqués par les « souvenirs écran » ou les « souvenirs indifférents ». Relâchant ou abandonnant les mécanismes de protection du Moi, l'individu va alors pouvoir « tourner la page » pour « redevenir lui-même ${ }^{19}$ ".

Pendant les années de rééducation à la campagne, les cinéastes passent leur temps de jeunesse parmi les gens les plus défavorisés de la société et témoignent personnellement des dures conditions de vie et de la pauvreté des paysans. Leur expérience d'«exilé » à la campagne nourrit l'inspiration créative des cinéastes et sollicite leur réflexion sur la culture traditionnelle. Du coup, la Cinquième Génération fixe ses caméras sur les territoires pauvres, l'existence misérable des paysans, comme dans La Terre jaune (Chen Kaige, 1984) et Le Sorgho rouge (Zhang Yimou, 1988), films représentatifs des deux grands maîtres de la Cinquième Génération. D’après Luisa Prudentino, dans le film La Terre jaune, la dénonciation des effets néfastes de la Révolution culturelle est violente : non seulement elle n'a rien apporté à la vie du peuple chinois, en laissant vivre les paysans dans des conditions de pauvreté et de sous-développement accablantes, mais elle a aussi détruit les valeurs traditionnelles ${ }^{20}$. Au lieu de mettre en scène directement la tragédie de la Révolution culturelle, les cinéastes portent un regard critique et mènent une réflexion sur les crises sociales avec un intérêt particulier porté à l'existence misérable dans les régions rurales et la terre qui est métaphorique de la tradition et des racines de l'homme. Pendant la Révolution culturelle, les jeunes instruits ont été exilés dans les régions rurales, où ils ont été marginalisés à la fois par la communauté rurale dans laquelle ils avaient de la difficulté à s'intégrer et par l'ordre urbain duquel ils s'éloignaient. C'est cette identité marginale qui permet aux cinéastes de se sortir de leur situation et d'avoir une perception objective de la calamité en tant qu'observateurs pour mener une réflexion approfondie sur cette histoire. Hantés par la réflexion profonde sur l'histoire et la tradition d'où le présent est issu, ils cherchent à remettre en exergue les valeurs traditionnelles détruites dans la Révolution culturelle et à remédier à la rupture en renouant le passé et le présent.

La prise de conscience de la valeur individuelle

Après avoir passé quelques années en rééducation à la campagne, les cinéastes sortent de la fièvre de l'idéalisme et du culte maoïste, et commencent à mener une réflexion sur la relation entre l'individu et l'institution, où s'engendre une prise de conscience de la valeur individuelle, qui constitue également un leitmotiv du cinéma de la Cinquième Génération. On trouve à ce sujet les films La Terre jaune, La grande parade et Le Roi des enfants, qui constituent la trilogie de Chen Kaige. Dans La Terre jaune, l'individu est pour la première fois représenté en tant que figure rebelle contre des rites et une culture féodale. L'héroïne Cuiqiao est construite au début du film comme une figure dépossédée de caractère proprement individuel : obligée de se soumettre aux ordres de son père, elle doit se marier avec un homme plus âgé qu'elle. Tandis que l'arrivée du soldat Guqing 
ravive ses espoirs, elle désire sortir des contraintes des rites féodaux et chercher son bonheur en fuyant. La jeune fille sacrifie enfin sa jeune vie. Ainsi, la prise de conscience de l'individu est construite par le cinéaste d'une manière implicite. Dans son deuxième film, $L a$ Grande parade, Chen aborde la tension entre l'individu et l'institution en racontant l'entraînement militaire auquel se soumettent quatre-cents jeunes soldats se préparant pour les célébrations du trente-cinquième anniversaire de la fondation de la République Populaire de Chine. Afin de participer à ce grand événement au cours duquel la troupe défilera sur la Place Tiananmen, les jeunes soldats paient un prix afin de garder leur place dans le défilé : l'un renonce à assister aux obsèques de sa mère ; un autre aux jambes tordues se soumet à des tortures nocturnes pour les redresser. D'après Luisa Prudentino, l'armée est ici une métaphore efficace de la société qui impose l'uniformité et écrase toute velléité d'initiatives individuelles : les institutions ont fini par étouffer l'individualisme ${ }^{21}$. L'interprétation de Prudentino est pertinente. L'armée fait allusion à la collectivité et au système du mécanisme institutionnel qui impose les valeurs collectivistes, étouffe l'individu et supprime l'individualité et les sentiments individuels, mécanisme sur lequel le film porte de manière implicite un regard critique. Sous le système de la machine d'état tout puissante, l'individu n'est plus qu'une proie.

Pour Chen Kaige, la dénonciation de la catastrophe de la Révolution culturelle est beaucoup plus explicite dans le troisième film Le Roi des enfants. Le film raconte l'expérience d'un jeune instruit qui enseigne dans un village après avoir passé plusieurs années en « rééducation » à la campagne. Après avoir découvert que l'ancien enseignant demandait aux élèves de recopier un texte sans le comprendre, le jeune instituteur propose une méthode différente en leur apprenant le sens de chaque caractère et en les encourageant à penser indépendamment. Pourtant, cette nouvelle approche des méthodes d'enseignement provoque le mécontentement des autorités. Du coup, le jeune instruit est finalement expulsé de l'école et retourne aux camps de travail. D'après Zhang Xudong, le film Le Roi des enfants est tout d'abord un travail autobiographique moulé dans une allégorie. Chen construit, à travers ce jeune intellectuel envoyé comme enseignant dans une école dans des montagnes reculées, l'allégorie de toute sa génération, à la recherche d'une mémoire collective qui puisse donner raison à l'identité personnelle ${ }^{22}$. $\mathrm{Au}$ lieu de mettre en accusation de façon explicite le mouvement d'envoi des jeunes instruits à la campagne, le cinéaste tente de mener une réflexion profonde sur la dictature politique qui détruit la possibilité de prise d'initiatives de la part des individus et impose inéluctablement un contrôle de la pensée à l'individu.

La réflexion sur le destin individuel

C'est dans le cinéma de la Cinquième Génération que le destin individuel est pour la première fois au cœur de la réflexion des cinéastes. Le film de Tian Zhuangzhuang fait référence, de façon indirecte, à la période de la Révolution culturelle. À la différence de ses contemporains qui s'intéressent aux sujets ruraux, Tian porte un intérêt particulier à la vie des gens des minorités ethniques et décrit leurs rituels et leurs expériences. Cependant, Tian indique que le but de son cinéma reste le même que celui des autres réalisateurs de son époque, c'est-à-dire de « faire passer un message très clair sur la société chinoise contemporaine ${ }^{23}$ ». Pour bien comprendre ses films, il faut en décoder les métaphores et les signaux. Par exemple, dans son premier long métrage La Loi du terrain de chasse (1984), le terrain de chasse, selon Luisa Prudentino, symbolise la société chinoise après ce véritable massacre qui dura dix $a^{2}{ }^{24}$. L'action de chasse fait allusion à la lutte des classes et à la persécution entre les gens pendant la Révolution culturelle, qui semble 
tout aussi cruelle et sauvage que la chasse. C'est ce que le réalisateur Tian Zhuangzhuang révèle, au début de la Révolution culturelle, les gens étaient extrêmement cruels les uns envers les autres, comme s'ils avaient perdu une certaine humanité. Dans La Loi du terrain de chasse, la façon dont les animaux sont chassés semble être très cruelle et très sauvage alors que les scènes filmées dans les prairies semblent calmes. Au contraire, la relation entre les êtres humains est beaucoup plus terrifiante ${ }^{25}$.

Dans le deuxième long métrage de Tian, Le Voleur de chevaux (1986), comme dans le premier long métrage, le réalisateur assoit son point de vue sur les crises sociales de la société chinoise à l'aide de la représentation de l'expérience des gens issus des minorités ethniques. Le réalisateur retrace cette fois la vie quotidienne des Tibétains et raconte l'histoire de Norbu, un pâtre exclu de son clan du fait qu'il gagnait sa vie en volant des chevaux. Norbu mène désormais une vie d'exilé, misérable, avec sa famille suite au bannissement. Pour être admis de nouveau par son clan, il renonce au vol de chevaux et vend son cheval pour vivre. Après avoir subi une série de malheurs, il doit toutefois se remettre à voler des chevaux. Il se sacrifie finalement afin de protéger sa famille. D'après Luisa Prudentino, le but du réalisateur consiste à « montrer que personne n'a pu échapper aux horreurs de la Révolution culturelle, que cette expérience a également été traumatisante pour les minorités ethniques dont on ne parle jamais ${ }^{26} »$. Nous remarquons que dans ses deux films, Tian Zhuangzhuang est obsédé par le sujet des ethnies minoritaires et notamment par le destin de l'individu au sein d'une communauté minoritaire qui impose des contraintes et des règles. En métaphorisant l'ordre institutionnel par le clan minoritaire et en montrant la faiblesse de l'individu face aux contraintes de l'institution, le réalisateur tente de faire réfléchir les gens sur leur attitude vis-à-vis de la révolution maoïste, qui peut être dans un certain sens interprétée comme une religion politique et un culte aveugle.

La quête mémorielle de l'histoire de la Révolution culturelle dans le cinéma des cinéastes de la Cinquième Génération ne pourra s'effectuer sans tenir compte de leur identité de jeunes instruits « exilés ». Leur mémoire de la Révolution culturelle et leur identité de jeunes instruits s'épousent l'une l'autre et se fondent mutuellement pour produire un récit collectif et édifier un mythe d'une génération spécifique. Dans le cinéma, les souvenirs personnels des cinéastes prennent part à la construction du récit collectif de l'Histoire par l'apport d'expériences personnelles et donnent à l'épopée de ce groupe la dimension singulière de l'expérience personnelle. Ainsi les formes individuelles de la mémoire et de l'identité témoignent du passage à des formes collectives. Les cinéastes évoquent cette expérience consciemment à travers les métaphores, par lesquelles ils mènent une critique et une réflexion profonde sur le système tyrannique et le suivisme aveugle pendant la Révolution culturelle, événements qui ont détruit les valeurs individuelles et mis tout destin individuel en péril. Là, les cinéastes de la Cinquième Génération retrouvent leur rôle et leur responsabilité en tant qu'artistes humanistes. Suite aux ruptures et au nihilisme de la Révolution culturelle, s'impose à eux le devoir de transmettre la culture traditionnelle et la mémoire, de réévaluer l'histoire et de mener une réflexion profonde sur cette dernière afin de s'interroger sur la place de l'individu dans l'histoire. Revenir sur le passé permet de tenir ensemble les trois dimensions temporelles - le passé, le présent et le futur et de réfléchir sur ce qui a été fait et ce qui pourrait être fait dans le futur, afin de renouer une continuité. Les souvenirs que les cinéastes de la Cinquième Génération gardent de cette histoire permettent qu'une 
identité collective de " génération de réflexion » se nourrisse et se fonde, et que le sentiment de leur identité se perpétue.

\section{APPENDIXES}

Les coordonnées de l'auteure :

Adresse professionnelle : Bureau A03, Institut de recherche sur les industries culturelles, Université de Shenzhen, $n^{\circ} 3688$ Avenue Nanhai, Shenzhen, Province du Guangdong, Chine

Email : dengwenjun917@gmail.com

\section{NOTES}

1. Gian Piero Brunetta, «Le cinéma », dans Mario Isnenghi (dir.), L'Italie par elle-même. Lieux de mémoire italiens de 1848 à nos jours, Presses de l'Ecole normale supérieure, 2006, p. 218.

2. Ibid, p. 219.

3. Michel Bonnin a consacré un ouvrage intitulé Génération perdue : le mouvement d'envoi des jeunes instruits, dans lequel, il écrit, à l'égard de la définition de cette " génération perdue ", « ce sont en principe les trois promotions de collégiens et de lycéens des années 1966-1968, on les appelle 'les trois vieilles promotions'. Les membres de ces "trois vieilles promotions" sont ceux qui ont le plus fort sentiment d'appartenance à un groupe spécifique, car leur expérience est la plus marquante: participation à la Révolution culturelle comme Gardes rouges, puis départ quasi général à la campagne dans des conditions souvent très dures et séjour particulièrement long puisque certains ont dû attendre dix ou onze ans pour revenir en ville, à la fin du mouvement, en $1979 »$.

4. Les cinéastes de la Cinquième Génération réalisent une série de films qui mènent une réflexion sur l'histoire et la culture, tels que Un et huit (Zhang Junzhao, 1983), La Terre jaune (Chen Kaige, 1984), Bloodshed in the Dark Valley (Wu Ziniu, 1984), La Loi du terrain de chasse (Tian Zhuangzhuang, 1985) , L'incident du canon noir (Huang Jianxin, 1986), Le Voleur de chevaux (Tian Zhuangzhuang, 1986), La Grande parade (Chen Kaige, 1986), Le Roi des enfants (Chen Kaige, 1987), Le Sorgho rouge (Zhang Yimou, 1988), The Evening Bell (Wu Ziniu, 1988), etc.

5. Joël Candau, Mémoire et identité, Paris, PUF, 1998.

6. Ibid, p.10.

7. Ibid, p.9.

8. L'autobiographie de Chen Kaige a été publiée premièrement sous le titre de «Ma vie comme garde rouge" par les Éditions Kodansha du Japon en 1989 alors que le titre chinois était "Shaonian Kaige» (Jeune Kaige); Wo de qingchun huiyi lu (Ma jeunesse) publié en 2009 est un remake de ce dernier.

9. Chen Kaige, Wo de qingchun huiyilu (Ma jeunesse), Pékin, Renmin daxue chubanshe (Éditions de l'Université du peuple), 2009, p.2.

10. Ibid.

11. Tony Rayns, King of the Children: And the New Chinese Cinema, London, Faber and Faber, 1989, p.

43. 
12. Ibid, p.45.

13. Liu Weihong, "Yu Wu Ziniu tan Wu Ziniu» (Parler de Wu Ziniu avec Wu Ziniu), Dangdai Dianying (Cinéma contemporain), $\mathrm{n}^{\circ}$ 4, 1988, p. 110.

14. Ibid.

15. Joel Candau, Mémoire et identité, Paris, PUF, 1998, p. 136.

16. L'Académie militaire de Huangpu était un institut de formation militaire fondé en 1924 où ont été formés de nombreux officiers qui sont devenus célèbres pendant la guerre sino-japonaise et la guerre civile chinoise.

17. Le Kuomingtang, un parti politique de la République de Chine créé par Sun Yat-sen, domina le gouvernement central de la République de Chine à partir de 1928 jusqu'à la prise de pouvoir des communistes en 1949 ; son influence ne se limite aujourd'hui qu'à Taiwan.

18. Maurice Halbwachs, La mémoire collective, Paris, Éditions Albin Michel, 1994 ; réédition de l'ouvrage du même titre, Paris, Éditions PUF, 1968.

19. Joel Candau, Mémoire et identité, Paris, PUF, 1998, p.56.

20. Luisa Prudentino, Le regard des ombres, Paris, Bleu de Chine, 2003, p.28

21. Luisa Prudentino, Le Regard des ombres, Paris, Bleu de Chine, 2003, p.29.

22. Zhang Xudong, Chinese Modernism in the Era of Reforms-Cultural Fever, Avant-Garde Fiction, and the New Chinese Cinema, Duke University Press Books, 1997.

23. Lusia Prudentino, Le Regard des ombres, Paris, Bleu de Chine, 2003, p.34.

24. Ibid.

25. Ibid.

26. Ibid. p.35

\section{AUTHOR}

\section{WENJUN DENG}

Post-docteure actuellement à l'Institut de recherche sur les industries culturelles, Université de Shenzhen, Chine. Axe de recherche actuel : les industries créatives et culturelles ; Ancienne docteure en histoire culturelle à l'école doctorale Arts et Médias, Université Paris 3 ; son orientation de recherche consiste en les questions de mémoire et identité, et la représentation des traumatismes à l'écran. Sa thèse, intitulée « La mémoire collective de la Révolution culturelle dans le cinéma chinois contemporain », a été soutenue le 14 juin 2013 avec la mention « Très honorable avec félicitations ». 